\title{
OCUPACIÓN ANTRÓPICA Y RIESGOS DE INUNDACIÓN EN LA LLANURA DEL RÍO GUADALQUIVIR (SECTOR PALMA DEL RÍO-SEVILLA, ESPAÑA)
}

\author{
Rafael Baena Escudero \\ Inmaculada Guerrero Amador \\ Belén García Martínez \\ J. Carlos Posada Simeón \\ Departamento de Geografía Física y Análisis Geográfico Regional. Universidad de Sevilla \\ baena@us.es, inmaguer@us.es,mbelen@us.es, posada@us.es
}

\section{RESUMEN}

Se presenta la ocupación antrópica de la llanura de inundación del Guadalquivir desde época histórica. Con ello se pone de manifiesto la falta de una planificación integrada frente al riesgo de inundación de la misma desde mediados del siglo XX hasta la actualidad. Esta situación genera cambios geomorfológicos irreversibles en el cauce y un comportamiento diferenciado en el riesgo potencial de inundación. En la ciudad de Sevilla, las intervenciones estructurales unidas a la incisión del cauce permiten evacuar las crecidas para magnitudes inferiores a $3.500 \mathrm{~m}^{3} / \mathrm{s}$. Aguas arriba de Cantillana, la colmatación del cauce vinculada a la presa, la convergencia de afluentes regulados y la expansión urbana, provocan en la actualidad riesgos de inundación en zonas históricamente no inundables para estos caudales.

Palabras clave: llanura de inundación, ocupación antrópica, riesgos de inundación, río Guadalquivir, España.

Fecha de recepción: noviembre 2014.

Fecha de aceptación: noviembre 2015. 


\section{ABSTRACT}

We present the anthropic occupation of the flood plains of the Guadalquivir River from historic times. With it, we illustrate the lack of an integrated plan that would defend it from the risk of a flood from the middle of 20th century until today. This situation creates irreversible geomorphological changes in the riverbed and a differenciated behavior in the potential risk of flooding. In Seville, the structural works together with the incision of the riverbed permit the draining of the flooding of magnitudes lower than $3.500 \mathrm{~m}^{3} / \mathrm{s}$. Up the river from Cantillana, the filling of the riverbed linked to the damn, the confluence of the regulated tributaries and the urban spreading, provoke today risks of floods in historically non-floodable areas for these water flows.

Keywords: Flood plain, anthropic occupation, risks of flood, Guadalquivir River, Spain

\section{INTRODUCCIÓN}

Las llanuras aluviales constituyen espacios, por lo general, bien individualizados en los que se plasma el equilibrio dinámico (balance incisión-colmatación) alcanzado por el hidrosistema fluvial a lo largo de decenas de miles de años (Baena, 2006). Cumplen, por tanto, una función natural de respuesta a las condiciones de flujo de los ríos que encuentra su máxima expresión durante los momentos de crecida cuando actúan como áreas de laminación y decantación sedimentaria (Ward, 1978; Brierley \& Fryirs, 2004).

Pese a estos condicionantes de carácter físico y al riesgo que este tipo de espacios entraña, sin embargo el hombre los ha considerado lugares privilegiados para la implantación de sus actividades, convirtiéndolos desde hace más de 2.000 años, en los grandes ejes contemporáneos de articulación y desarrollo económico del territorio (Tockner \& Stanford, 2002; Bravard, 2006). Ello ha supuesto modificaciones importantes en la dinámica fluvial e irreversibles en la morfohidrología de las llanuras de inundación de los ríos (Macklin \& Lewin, 2003; Bravard, 2004; Bescós y Camarasa, 2004). Esta situación alcanza su máxima expresión durante el final del siglo XX cuando, impulsados por un elevado grado de desarrollo tecnológico en el control de los fenómenos hidrológicos naturales (regulación de cuencas, diques y modificaciones del cauce) y ciertos eventos climáticos (sequías prolongadas), los países desarrollados se lanzan a un aprovechamiento integral de las mismas (intensificación agrícola, extensión de las zonas industriales y urbanas, implantación de infraestructuras, etc.) no considerando, en la planificación territorial, dinámicas fundamentales que rigen estas unidades geomorfológicas (Baena et al., 2004; Langhammer \& Vilimek, 2006).

El resultado ha supuesto fuertes alteraciones en los principales hidrosistemas fluviales europeos en cuyos valles más importantes y pese a las experiencias adquiridas a finales del pasado siglo (Ramos, 1983; Roux et al., 1989; Garzón et al., 1990; Ollero, 1991; Klingeman et al., 1994; Peiry et Nouguier, 1994; Guerrero y Baena, 1998), aún se continúan ejecutando intervenciones en cauces y riberas de cara a la ocupación del territorio (Ibizate, 2006; Guerrero et al., 2014). Todo ello ha ocasionado la aparición de cambios geomorfológicos irreversibles en los cauces y un comportamiento diferenciado en el riesgo potencial de inundación según las zonas de las llanuras de inundación (Baena et al., 2006). 
En este estudio se presentan los rasgos más destacados de la geomorfología fluvial de la llanura aluvial del río Guadalquivir en la provincia de Sevilla con especial atención a sus principales elementos morfohidrológicos y a la función de estos durante las inundaciones. Como objetivos específicos se pretende realizar una evaluación de la ocupación histórica de la llanura de inundación así como de su grado de intervención durante los últimos 50 años. Esto nos permitirá comprender la dinámica espacial y temporal de los cambios geomorfológicos acontecidos en el cauce y en las zonas inundables de la llanura. Igualmente se presenta la funcionalidad actual de la llanura del bajo Guadalquivir frente a eventos recientes de inundación.

\section{II. ÁREA DE ESTUDIO, METODOLOGÍA Y FUENTES}

El río Guadalquivir, con una aportación anual de 7.230 $\mathrm{Hm}^{3}$ y una cuenca hidrográfica de $57.527 \mathrm{Km}^{2}$ (Confederación Hidrográfica del Guadalquivir, 2012), representa el principal colector del Sur de España. Su régimen de tipo pluvial subtropical (Masachs, 1954), está claramente influenciado por la llegada de frentes asociados a las borrascas atlánticas durante los meses de Noviembre a Marzo, lo que le proporciona una extremada variabilidad tanto estacional $(\mathrm{Cq}=13,8)$ como interanual $(\mathrm{Cq}=7,9)$. El caudal modular del Guadalquivir en Sevilla es de unos $185 \mathrm{~m}^{3} / \mathrm{s}$ para un área de $49.979 \mathrm{Km}^{2}$, correspondiendo más del $42 \%$ a los afluentes que confluyen en la zona de estudio. Por su parte las inundaciones, con picos de crecida comprendidos entre los 1.500 y los $12.000 \mathrm{~m}^{3} / \mathrm{s}$, sólo dejan sentir sus efectos sobre las poblaciones del entorno a partir de caudales superiores a $\operatorname{los} 3.000 \mathrm{~m}^{3} / \mathrm{s}$ debido a las obras de encauzamiento y defensa (Vanney, 1970; Baena et al., 2006). El resultado ha sido la configuración desde el Tardiglaciar (Baena et al., 2014), de una amplia llanura aluvial por donde discurre el río con trazado meandriforme de alta sinuosidad (1,5). Dentro de él, coexisten meandros de amplios radios de curvatura heredados de finales del siglo XVIII, con otros de reducido tamaño debido a rectificaciones de trazado por acortamientos a inicios del siglo XX (García Martínez, 2003).

El área de estudio comprende una zona alargada de más de $80 \mathrm{~km}$ de longitud por unos 6 km de anchura media que, a ambos lados del río, se extiende entre la ciudad de Palma del Río situada en el límite con la provincia de Córdoba y la ciudad de Sevilla (Fig. 1). Dentro de ella el río presenta dos sectores denominamos estuarino y continental. EL primero, se extiende desde el mar hasta la presa de Alcalá del Río, coexistiendo en él la influencia mareal y la dinámica fluvial, esta última exclusiva sólo durante las crecidas; mientras que el segundo, a partir de esta presa, sólo presenta dinámica fluvial. Se trata de un territorio bien diferenciado respecto al resto de las comarcas naturales que lo rodean. Esto es, Sierra Morena al Norte y Noreste (borde meridional paleozoico del Macizo Hercínico); las campiñas al Oeste (margas y limos arenosos del Neógeno de la Cuenca del Guadalquivir); y las terrazas cuaternarias del Guadalquivir al Sur (gravas, arenas, arcillas y limos).

Para el estudio se ha contado con la cartografía geomorfológica del Bajo Guadalquivir (escala; 1:50.000) realizada a partir de fotogramas aéreos a escala 1:30.000 de 1956 (Baena, 1993), como base para la identificación de aquellos elementos morfohidrológicos fundamentales en el funcionamiento natural de la llanura aluvial (límites, zonas de inundación, cauces de crecida, meandros abandonados, escarpes de terrazas y afluentes). Al mismo tiempo, la evaluación de los cambios acontecidos como consecuencia de la intervención humana en los 
55 años considerados se ha realizado mediante trabajo de campo y la fotinterpretación de los fotogramas aéreos de 1956 (U.S. Army a escala 1:30.000), 1973 (CEFTA S.A. a escala 1:25.000) y 1981 (FOYCAR S.A. a escala 1:25.000). También se han considerado las ortoimágenes multitemporales, Landsat 5 de 1990 a escala 1:100.000 del Instituto Geográfico Nacional (M.O.P.U.) y SPOT de 1991 a escala 1:50.000 correspondientes al área metropolitana de Sevilla (Agencia de Medio Ambiente-Dirección General de Ordenación del Territorio, Junta de Andalucía); y ortofotografía digital en color de Andalucía de 2007 y 2010/2011 con resolución de 0,5 m (Consejería de Vivienda y Ordenación del Territorio. Instituto de Cartografía de Andalucía).

Figura 1

LOCALIZACIÓN DE LA ZONA DE ESTUDIO Y PRINCIPALES NÚCLEOS DE POBLACIÓN DE LA LLANURA ALUVIAL DEL GUADALQUIVIR EN LA PROVINCIA DE SEVILLA

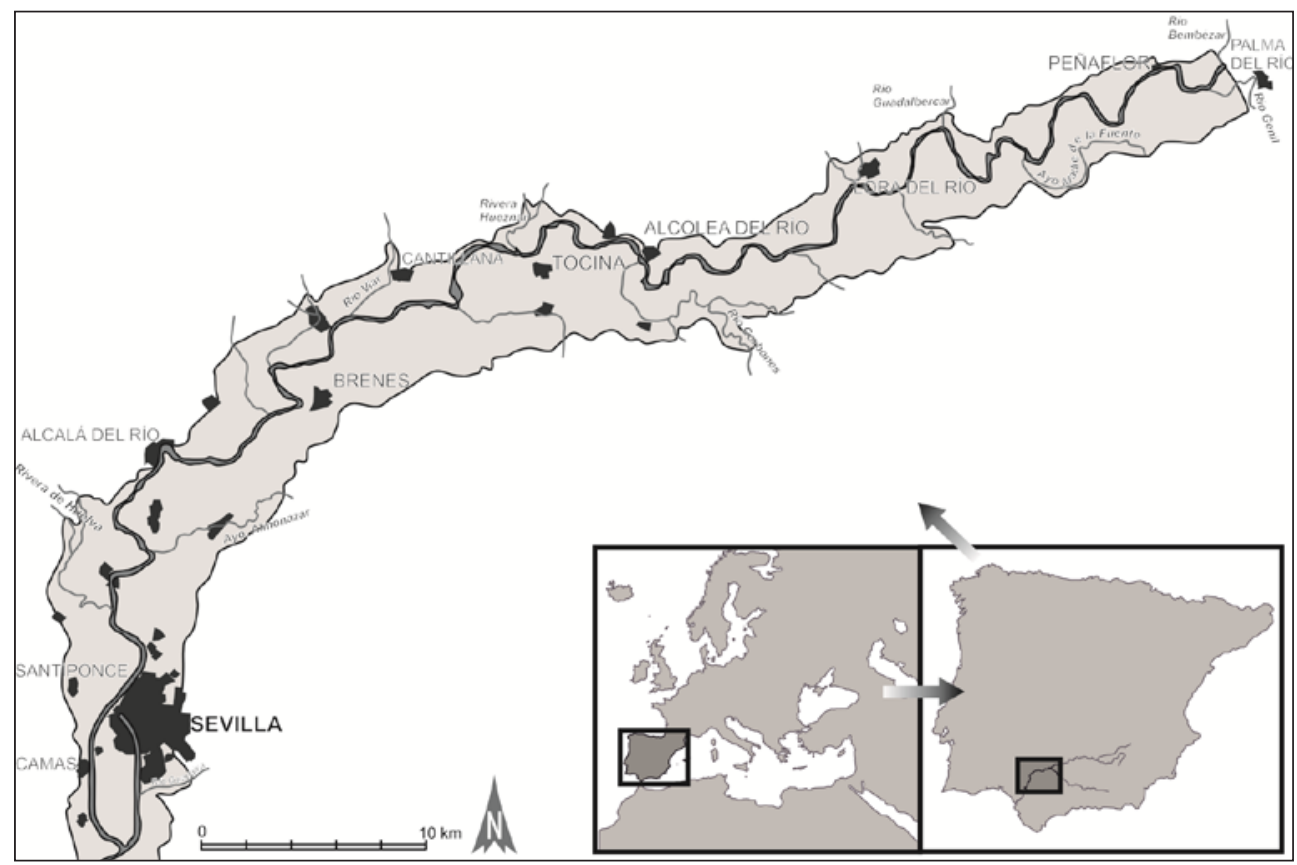

Elaboración propia.

Para el funcionamiento hidrológico extremo se ha tenido en cuenta el registro histórico de crecidas del río Guadalquivir, en su tramo bajo, mediante las referencias encontradas en distintas obras recopilatorias (Borja Palomo, 1878; Vanney, 1970; Ministerio de Obras Públicas y Urbanismo, 1985); mientras que para el correspondiente al siglo XXI, los datos proceden del Sistema Automático de Información Hidrológica del Guadalquivir -SAIH- en la presa de Alcalá del Río (Agencia Andaluza del Agua, 2011). Estos últimos han sido completados con los proporcionados por Guerrero y Baena (1998) y Baena et al., (2006) en cuanto al funcionamiento de la llanura de inundación durante las crecidas. 


\section{ELEMENTOS MORFOHIDROLÓGICOS Y FUNCIONAMIENTO NATURAL DE LA LLANURA ALUVIAL DEL GUADALQUIVIR EN LA PROVINCIA DE SEVILLA}

La llanura aluvial del Guadalquivir comprende el espacio de topografía plana y reducida altitud sobre el nivel del mar, entre 8 y $50 \mathrm{~m}$ para la provincia de Sevilla, que queda limitado en sus márgenes por importantes escarpes. Estos se desarrollan tanto sobre la última terraza media del Guadalquivir (T12), como sobre el sustrato de margas y limo-arenas que constituye el relleno neógeno de la Depresión (Baena, 1993). El río Guadalquivir discurre con una escasa pendiente longitudinal $(0,039 \%)$ y un trazado algo desplazado al norte, configurando un valle de sentido noreste-suroeste en su inicio y rumbo norte-sur desde Alcalá del Río hasta su desembocadura en el océano Atlántico (Fig.1). En esta llanura, al menos desde época romana, las crecidas han contado para su funcionamiento natural con múltiples elementos morfohidrológicos cuya importancia se detalla a continuación (Fig. 2).

Figura 2

PRINCIPALES ELEMENTOS MORFOHIDROLÓGICOS DE LA LLANURAALUVIAL DEL GUADALQUIVIR Y ÁREA INUNDADA DURANTE LA CRECIDA EXTRAORDINARIA DE 1963

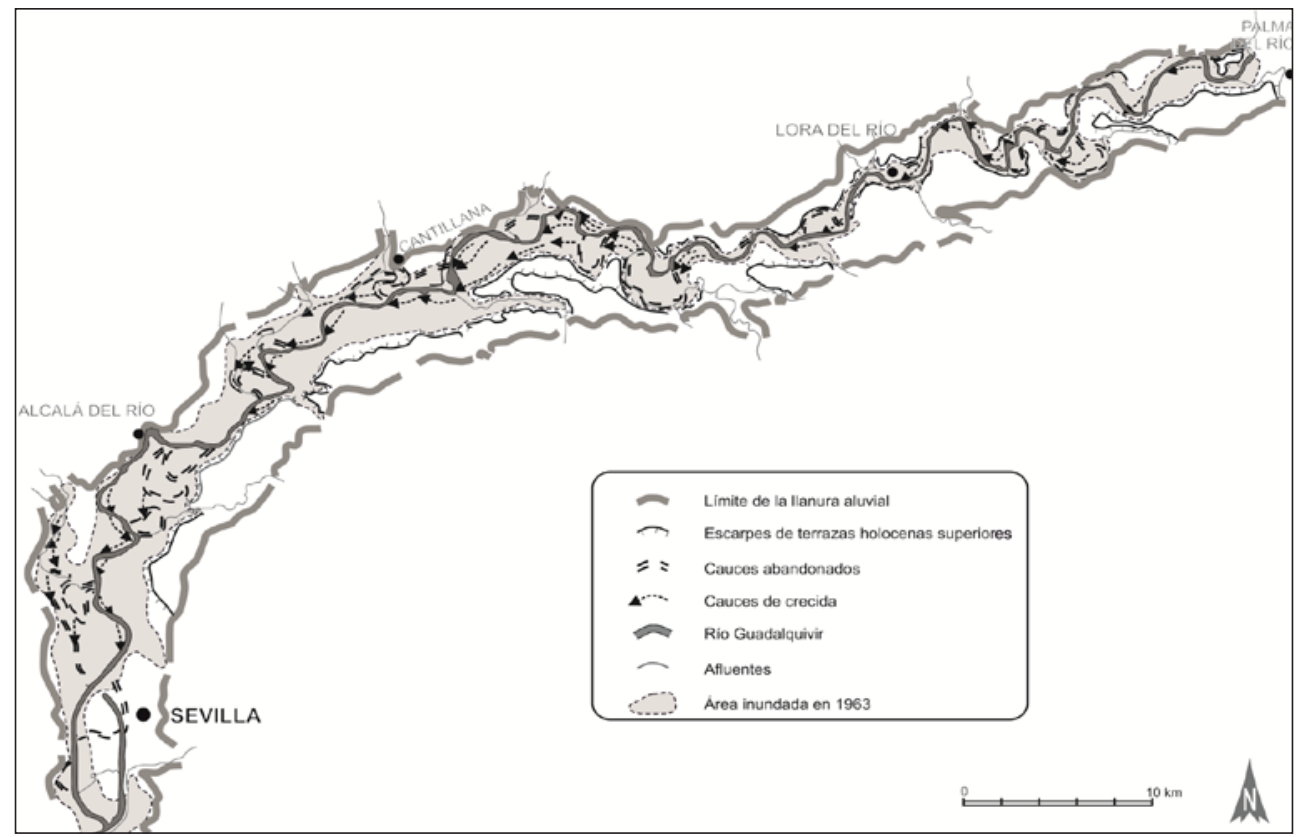

Fuente: Elaboración propia a partir de la cartografía geomorfológica del Bajo Guadalquivir (Baena, 1993).

\section{III.1. El río, su cauce ordinario y los afluentes}

Supone el factor principal en la conformación y evolución del valle, al tiempo que la principal discontinuidad espacial por el carácter permanente en la actualidad de su masa de agua. Esta discurre por un cauce ordinario único de entre 100 y $150 \mathrm{~m}$ de anchura media, 
y de 6 a 10 m de altura bajo el plano aluvial. En él, tienen lugar las frecuentes oscilaciones estacionales de caudal, con mínimos en septiembre $\left(32 \mathrm{~m}^{3} / \mathrm{s}\right)$ y máximos en primavera (300$400 \mathrm{~m}^{3} / \mathrm{s}$ ), sin que en ningún caso estos rebasen el estado de bankfull estimado en $900 \mathrm{~m} / \mathrm{s}$ (Vanney, 1970). De igual modo, destacan los cambios de pendiente en el lecho del río con un interesante punto de inflexión en el tramo comprendido entre Cantillana y Brenes, donde los valores de pendiente media se reducen drásticamente hasta alcanzar el 0,00044\% del tramo de estuario (Conf. Hidr. Guadalq., 1977). Es aquí donde sitúan las fuentes documentales de época romana la llegada del influjo de las mareas (Chic, 1979 crf., a Estrabon y Posidonio, s. I a. de C.). Como resultado, se ocasionan importantes variaciones en la velocidad de la corriente que generan un modelo meandriforme de orillas abruptas. En ellas la vegetación natural de ribera desempeña un importante papel, fijando los materiales limo-arenosos de la orilla y colonizando los sistemas de barras con especies riparias de alto valor ecológico.

En cuanto a los afluentes, igualmente numerosos en este sector de la llanura, presentan un funcionamiento tanto permanente como estacional según su recorrido y área drenada. Entre los procedentes de Sierra Morena destacan los ríos Retortillo, Guadalbarcar, Rivera de Huéznar, Viar y Rivera de Huelva. Todos ellos tienen fuertes pendientes, modestos caudales medios $\left(7,75 \mathrm{a} 12,2 \mathrm{~m}^{3} / \mathrm{s}\right)$ y extrema torrencialidad, lo que les hace muy peligrosos en sus efectos sobre la llanura al suministrar una importante carga de arrastre (gravas y arenas). Respecto a los afluentes de la margen izquierda, destaca el río Genil por su contribución decisiva a la regulación anual del Guadalquivir ( $40 \mathrm{~m}^{3} / \mathrm{s}$ de caudal medio), el descenso de la pendiente del río y el aumento en la amplitud de la llanura, configurando a partir de su desembocadura el denominado Bajo Guadalquivir (Baena, 1993). Otros afluentes importantes de esta margen son los ríos Corbones y Guadaira, ambos con caudales más moderados que los procedentes de Sierra Morena aunque con mayores magnitudes en sus crecidas (superiores a $500 \mathrm{~m}^{3} / \mathrm{s}$ ). Los afluentes suponen el principal factor en la dinámica hidrológica tanto del río como de su llanura. En el primer caso, aportan el $42,6 \%$ de su caudal final y una importante carga sedimentaria; mientras que en el segundo originan un serio obstáculo a la circulación del agua por el cauce principal. Se crean así, las condiciones necesarias para la fuerte divagación de los meandros, con frecuentes abandonos de cauces detectables a nivel histórico (García Martínez et al., 1999).

\section{III.2. Cauces abandonados, terrazas holocenas y llanura de inundación}

Corresponden a antiguos meandros o brazos abandonados por la corriente principal del Guadalquivir durante los momentos de crecida. Sobre el terreno sus trazados se identifican al constituir someras depresiones alargadas de entre 1 y 1,5 m por debajo de la topografía general. Junto al cauce funcional desempeñan un importante papel de cara a la circulación del agua en la llanura, actuando durante los momentos de crecida, como aliviaderos para evacuar los excedentes o, al menos, disipar su energía. La mayoría de ellos se remontan en su funcionamiento a época romana (s. I d.C.) según los numerosos restos arqueológicos encontrados en sus rellenos a entre 8 y 10 m de profundidad en el sector de estuario (Baena y Guerrero, 2009), o en sus márgenes en el sector continental aguas arriba de Alcalá del Río (Ponsich, 1974). Hasta mediados del siglo pasado su presencia era generalizada en todo el ámbito de la llanura aluvial («madres o ríos viejos») donde, al margen de la explotación agrícola, funcionaban como drenajes de los afluentes del Guadalquivir en sus tramos finales y como aliviaderos para los excedentes de riego. 
Por su parte las terrazas, conforman un sistema de 5 niveles cuyos escarpes delimitan las áreas de la llanura que son inundadas en función de la magnitud de la crecida. En la zona de estudio se agrupan en dos conjuntos morfogenéticos (Baena et al., 2014): las superiores de cronología Pleistoceno Superior-Holoceno, a +10-13 m (T13), +9-10 m (TH1) y +7-8 m (TH2) respecto a la altura de la lámina de agua; y las inferiores (Th1, Th2 y Th3), por debajo de los $6 \mathrm{~m}$ respecto al río. Las primeras permanecen al margen de toda inundación a excepción de la TH2 que junto a las segundas, vinculadas a la dinámica histórica de los meandros, integran el dominio funcional de la llanura de inundación (García Martínez, 1996; Guerrero y Baena, 1998; García Martínez y Baena, 2008). Espacialmente, su desarrollo es desigual respecto a ambas márgenes del río, concentrándose en mayor número sobre la izquierda. A veces, cómo sucede aguas abajo de la desembocadura del Genil o entre Tocina y Sevilla, las terrazas superiores (T13 y TH1) se adentran en la llanura actuando a modo de diques que dificultan y desvían la evacuación de las aguas desbordadas (Fig. 2).

En suma, la llanura aluvial del Guadalquivir en su tramo medio y sobre todo en el bajo, desempeña una función de zona de transferencia entre el drenaje generado por una superficie de cuenca de $49.979 \mathrm{~km}^{2}$ hasta Sevilla y una zona de carácter estuarino constituida por el entorno marismeño previo a la desembocadura. Ello, unido a la particular configuración «en espina de pez» de la red de drenaje, y al efecto represa de las terrazas holocenas y la marea, ocasionan una rápida concentración y obstrucción de los caudales de crecida que, a veces, llegan a colapsarla.

\section{LA OCUPACIÓN HISTÓRICA DE LA LLANURA ALUVIAL Y SU ESTADO DE INTERVENCIÓN A MEDIADOS DEL SIGLO XX}

Corresponde a época romana el comienzo de la ocupación generalizada de la llanura del Guadalquivir en relación con su elevada potencialidad agrícola y las posibilidades del río para la navegación (Bonsor, 1931; Chic, 1990). Ello supuso las primeras actuaciones antrópicas sobre el cauce destinadas a su regulación (diques de ribera y presas de laminación) y a la protección de las incipientes infraestructuras (puertos, calzadas, alfares, etc.). De igual modo, también tuvieron lugar los primeros impactos antrópicos sobre las riberas que destruyeron la cubierta forestal para extender los usos cerealísticos y olivareros. Pese a todo, el mundo romano evitó la construcción de grandes asentamientos poblacionales más allá de la terraza superior (T13), como límite natural de las inundaciones más frecuentes de la época. Se explica así el emplazamiento por encima de la cota de los 10-12 m respecto al río de la mayoría de las ciudades y pueblos de la vega: Sevilla (Hispalis), Santiponce (Itálica), Alcalá del Río (Ilipa Magna), Cantillana (Naeva), Alcolea del Río (Canania), Lora del Río (Axati) o Peñaflor (Celti).

Durante época medieval y moderna, la situación no cambió mucho salvo por la aparición de los primeros núcleos de población plenamente insertos en la llanura de inundación. Ello, además de indicar un descenso en la frecuencia de las inundaciones durante ese periodo (García Martínez y Baena, 1997), vincula su origen a la dependencia directa del agua del río para su empleo en la ganadería (abrevaderos, vados de cañadas, carboneo de los bosques de ribera, etc.) y la agricultura (extracción de aguas para el riego, molinos y «aceñas», etc.). Se configuran así poblaciones de origen medieval (s.s. XII-XIII) como La Algaba, La Rinconada, Brenes o Tocina al tiempo que surgen las primeras demarcaciones territoriales del ámbito aluvial («Cartas Puebla», «Libro de Repartimientos») que dan lugar a los términos municipales actuales. 
Ya a finales del siglo XVIII y durante todo el siglo XIX, con motivo de la falta de calado del río para la navegación y el incremento en el número de inundaciones, se emprenderán las primeras grandes obras en la llanura destinadas a su transformación. En un primer momento, éstas se centraron en la construcción de cauces artificiales o «cortas» de meandros aguas abajo de Sevilla (1795, «Corta de La Merlina»; 1816, «Corta Fernandina»; 1888, «Corta de Los Jerónimos»; 1926, «Corta de Tablada» y «Canal de Alfonso XII»; 1950, «Corta Punta del Verde»), para ya durante el siglo XX, completarse con nuevos trabajos encaminados a la regulación de la cuenca vertiente; la construcción de los primeros pasos permanentes sobre el río («Puente de Triana», 1852; «Puente de Palma del Río», 1885; «Puente de La Borbolla en La Algaba», 1923; «Puente de Alfonso XIII», 1926; «Puente de Lora del Río», 1930; «Puente de San Telmo», 1931; «Puente de San Juan de Aznalfarache», 1933; etc.); la laminación de la corriente de cara a la infructuosa navegación del Guadalquivir hasta Córdoba («Presa y Esclusa de Alcalá del Río», 1930 y «Cantillana», 1956; «Esclusa de Tablada», 1951); y la puesta en regadío de la llanura mediante presas y canales de derivación («Presa de Peñaflor», 1921; «Canal del Bajo Guadalquivir», 1909-1933 y «Canal del Viar», 1931-1934).

Se alcanza así la situación de mediados del siglo XX (Fig. 3, 1956), con una llanura profundamente transformada en la que destacan como más relevantes, las siguientes actuaciones por parte del hombre:

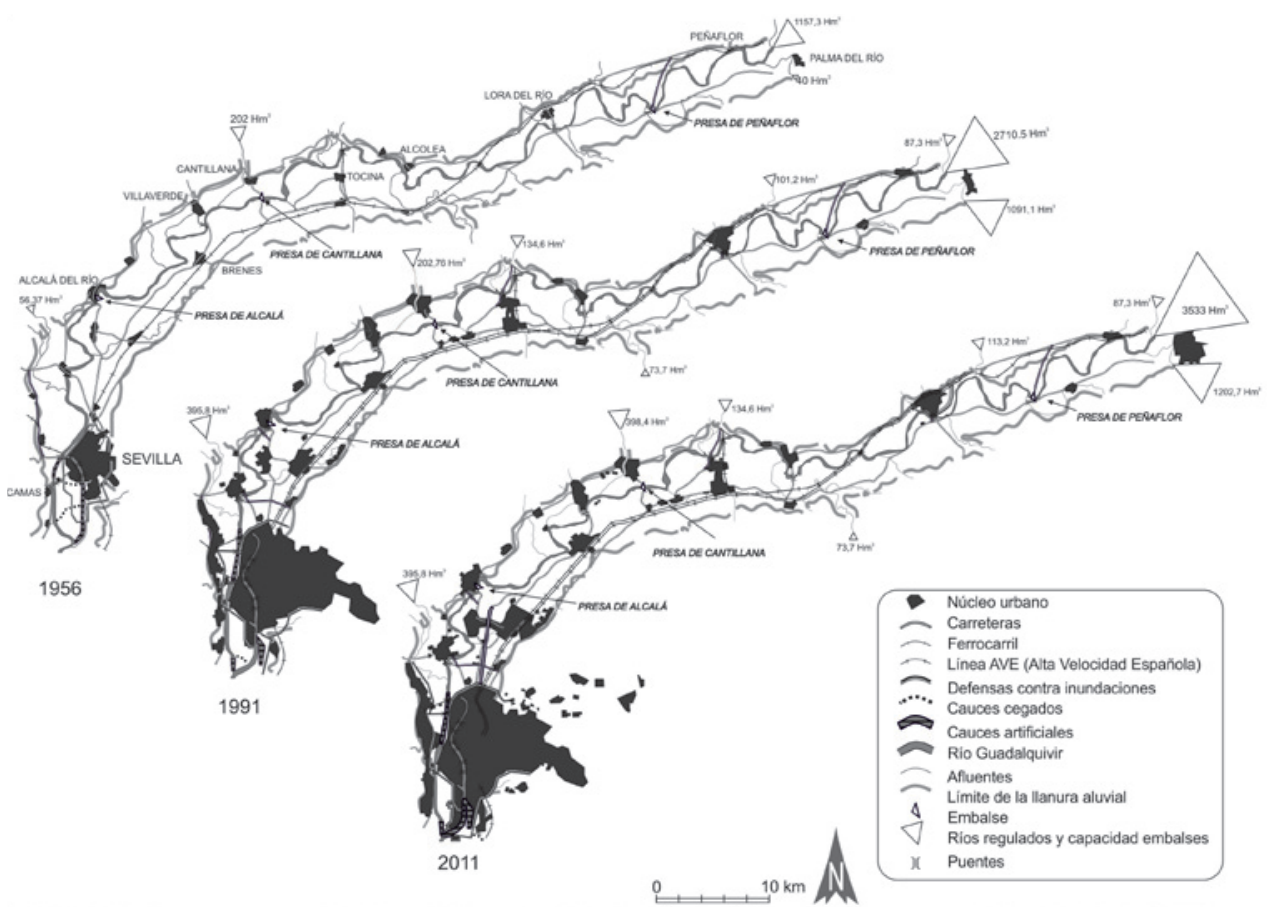

Fuente: elaboración propia. 


\section{IV.1. Intervenciones a nivel hidrológico}

Las cortas efectuadas redujeron más de $40 \mathrm{Km}$ el eje fluvial aguas abajo de Sevilla y los continuos dragados del río regularizaron su pendiente. Además, el desplazamiento artificial del río hacia la margen derecha en Sevilla consiguió dejar el cauce antiguo como dársena portuaria en el interior de la ciudad. Al mismo tiempo se contaba ya con 3 embalses sobre el cauce del Guadalquivir (Peñaflor, Alcalá del Río y Cantilla) y se había logrado la regulación parcial de afluentes como: el río Rivera de Huelva, Viar y Genil, con 298,37 Hm³ de capacidad, mediante la construcción de 5 embalses. Sobre el resto de la cuenca hidrográfica situada aguas arriba, el número de presas ascendía a 13, con una capacidad de 1.157,3 $\mathrm{Hm}^{3}$ (MOPU, 1991). Junto a esta obra hidráulica, la primera mitad del siglo XX supondrá la construcción de la mayor parte de las infraestructuras necesarias para la puesta en regadío de toda la vega aluvial. Esta intervención supuso uno de los factores clave en la organización del territorio, donde el entramado de canales y acequias de desagüe, ocupó la totalidad de la llanura y afectó a todos sus elementos morfohidrológicos.

\section{IV.2. Intervenciones a nivel de poblamiento, infraestructuras viarias y usos del suelo}

Sobre la llanura se asentaba una población de 461.185 habitantes en 1950 (Instituto de Estadística de Andalucía, 1992), repartidos entre 21 núcleos de población pertenecientes a 18 términos municipales de la provincia de Sevilla. Es entonces, cuando este espacio adquirió las directrices fundamentales en cuanto a su organización y articulación con el contexto regional, destacando la elevada densidad de carreteras y el trazado del ferrocarril. Un total de 10 puentes, 3 de ellos para ferrocarril, y 3 presas con travesía para vehículos, garantizaban un mínimo de comunicación entre las orillas del río en más de $110 \mathrm{~km}$ de recorrido. La mayoría de los puentes eran metálicos con uno o dos pilares centrales y siempre sobreelevados de 2 a $3 \mathrm{~m}$ por encima del cauce. Por su parte, la calidad de los suelos unida a la dotación de infraestructuras para el regadío, convirtieron a toda la llanura en un área de producción intensiva con más de 110.000 Ha regables. Esto supuso, al menos desde principios de siglo, la desaparición total de la vegetación natural con la excepción del degradado bosque galería de las orillas del Guadalquivir. En cuanto al uso urbano, aún quedaba restringido al entorno de los iniciales asentamientos salvo en Sevilla, donde el sistema de defensa perimetral contra-inundaciones, había permitido la expansión de la ciudad hacia la llanura de inundación (Fig. 3, 1956).

\section{LOS PRIMEROS CAMBIOS EN LA GEOMORFOLOGÍA FLUVIAL (AÑOS 50)}

El control hidrológico y las actuaciones sobre el río aguas abajo de Sevilla serán los inductores de los primeros cambios geomorfológicos que ya eran evidentes en los años 50. Estos afectaban desigualmente a los sectores de estuario y continental. En el primero porque las intervenciones para mejorar la navegación y evitar las inundaciones en el entorno de Sevilla repercutieron en: un aumento de la pendiente del río y su profundidad; la desaparición de las áreas de sedimentación en los meandros; una menor movilidad lateral del cauce; una disminución en la frecuencia de los desbordamientos; y una mayor y más rápida transmisión de los efectos de la marea. Se produce, por tanto, una transformación artificial del cauce que aumenta su anchura 
y profundidad y pierde entre un 30 y un $50 \%$ del espacio inundable en Sevilla. En el segundo porque aunque el río aún mostraba un estado seminatural, ya eran evidentes los efectos de la colmatación parcial en la presa de Alcalá del Río y también los de incisión aguas arriba hasta Cantillana. Desde aquí, la falta de embalses en los colectores procedentes de Sierra Morena permitía, aún en 1956, la llegada de abundante carga tractiva que se acumulaba en forma de barras centrales y laterales en el Guadalquivir. En cuanto a la llanura de inundación, se presentaba parcialmente obstruida en la zona por las infraestructuras ya mencionadas.

En esta situación, la llanura se vio sometida en febrero de 1963 a la última gran inundación de carácter extraordinario que ha tenido lugar en el Guadalquivir (Fig. 2). Estimada en 5.700 m³ s en el aforo de Alcalá del Río (Vanney, 1970), ocasionó la inmersión de las terrazas más bajas (TH2 e históricas), siendo sus efectos tanto más devastadores cuantas más obstrucciones encontró la punta de crecida a su paso. Destacan los daños en las poblaciones de: Peñaflor, Lora del Río, Alcolea, Tocina, Cantillana, Brenes, Alcalá del Río, La Algaba, La Rinconada y Sevilla; con un total de $1.097 \mathrm{Ha}$ inundadas y 15.316 personas evacuadas (M.O.P.U., 1985).

\section{EL CONTROL DEL HIDROSISTEMA DEL GUADALQUIVIR DURANTE LA 2 ${ }^{\mathrm{a}}$ MITAD DEL SIGLO XX}

Será a partir de 1960 y sobre todo de la avenida de 1963, cuando los organismos responsables de la gestión de la cuenca hidrográfica acometan un cambio en las prioridades de intervención hidráulica, hasta entonces orientadas al regadío y la mejora del puerto fluvial, dirigido al aumento en la regulación fluvial.

Por entonces, la capacidad de los embalses rondaba los $1.500 \mathrm{Hm}^{3}$, cantidad suficiente para alterar el régimen hidrológico del río pero no para su regulación completa, estimada entre 8.000 y $9.000 \mathrm{Hm}^{3}$ (Vanney, 1970). Los esfuerzos inversores realizados por el Estado durante los años 60 y 70 con la construcción de 12 embalses más $\left(655,32 \mathrm{Hm}^{3}\right)$, y los años 80 y 90 (13 embalses con 1.632,93 $\mathrm{Hm}^{3}$ ), quedaron lejos de alcanzar los valores teóricos necesarios para el control hidrológico total. No obstante, el haber cuadruplicado la capacidad de agua embalsada en los años 50, unido a las prolongadas sequías de inicios de los 80 y 90, crearon una falsa percepción de total eliminación de las crecidas catastróficas. Junto a ello, los planes de defensa contra inundaciones acometidos en el entorno de Sevilla desde 1975, aumentaron la sensación de seguridad frente a este tipo de riesgo tanto de la administración como de los ciudadanos. Esto explicaría la descoordinación existente entre las distintas administraciones, durante las crecidas de los últimos años, en relación con los episodios de inundación y la ordenación del territorio (Baena et al., 2006).

Así, tras la finalización del cauce hidráulico artificial denominado «Corta de La Cartuja» en 1982, se alejó definitivamente el río funcional de las inmediaciones de Sevilla. Además, la localización en la llanura de inundación de «La Exposición Universal» de 1992, permitió una nueva ordenación urbanística y la construcción de grandes infraestructuras viarias sobre el espacio aluvial que lo terminará configurando tal y como se muestra en los años 90 (Fig.3, 1991). Esto es con:

a) Una elevada concentración de población que alcanzaba en 1991 el 50,7\% del total de la provincia en tan sólo un 4\% de su territorio; lo que supone un 76,7\% más que a mediados de siglo con el consiguiente aumento del espacio edificado (Instituto de Estadística de Andalucía, 1992). 
b) Una alta densidad de infraestructuras viarias mejoradas respecto a las existentes a mediados de siglo (mayor anchura de firme, andenes, etc.) y completadas con la construcción de otras nuevas que bien circundan la ciudad de Sevilla o atraviesan la llanura aumentando la conectividad. Esto supuso un incremento en el número de puentes (superior a la docena), aunque ahora presentan menor altura sobre cauce y mayor sobreelevación del vial sobre la llanura. En cuanto al ferrocarril, se instaló una nueva línea de alta velocidad (AVE) que discurre paralela a la anterior pero con una mayor altura de firme e invadiendo, en ocasiones, la llanura de inundación.

c) Un sistema de diques contra las inundaciones, de entre 6 y $12 \mathrm{~m}$ de altura respecto al plano de inundación, que ahora se amplía por ambos lados del nuevo cauce en Sevilla. Así, la llanura de inundación en este sector quedó restringida a menos de $1 \mathrm{~km}$ de anchura.

d) Un aprovechamiento agrícola intensivo que, junto con la falta de recursos hídricos como consecuencia de la sequía, repercutió en un fuerte incremento en la explotación del acuífero aluvial conectado al río, estimado en 15 Hm³/año (Confederación Hidrográfica del Guadalquivir, 1977). Por otro lado, la falta de nuevos espacios para la agricultura impulsó a la ocupación de los cauces de crecida así como a la destrucción del bosque de ribera para su puesta en cultivo.

e) Una fuerte demanda de áridos para la construcción que a raíz de las numerosas obras de infraestructuras y el aumento en el sector urbano, supuso la extracción de gravas y arenas del propio cauce. Sobre todo, en el tramo continental, donde los aportes sólidos de los afluentes todavía permitían la formación de barras de acreción lateral dentro de él.

\section{EL AFIANZAMIENTO DE LOS CAMBIOS GEOMORFOLÓGICOS Y SUS REPERCUSIONES}

El mencionado control del hidrosistema del Guadalquivir generó, a finales del s. XX, una importante transformación del cauce y la llanura aluvial. En el primer caso, continuó de manera general la incisión y reducción de anchura del río. Este fenómeno se aprecia claramente en todo el tramo bajo continental al hacer el seguimiento diacrónico de las barras laterales del cauce que originan hasta dos nuevos niveles de terrazas en 30 años. Este proceso, progresivo y remontante a partir del tramo estuarino, presenta una dinámica particular en relación a la localización de las presas de este sector (Fig. 4):

a) En la presa de Alcalá del Río, la incisión se ve incrementada aguas abajo donde los taludes del cauce superan los $8 \mathrm{~m}$, mientras que aguas arriba se produce la colmatación inmediata a la misma.

b) En la presa de Cantillana, lo que predomina aguas abajo hasta Brenes, es la incisión con alturas de márgenes de entre 2 y $4 \mathrm{~m}$. Sin embargo aguas arriba, el efecto del nivel de base local de la presa, origina una fuerte colmatación del cauce (unos $2 \mathrm{~m}$ de media) que se extiende hasta Alcolea del Río.

c) En la presa de Peñaflor, la situación se repite con incisión aguas abajo hasta Alcolea del Río y colmatación en el cauce aguas arriba hasta las proximidades de Palma del Río. 
Figura 4

DINÁMICA DE INCISIÓN-COLMATACIÓN DEL RÍO GUADALQUIVIR VINCULADAAL EMPLAZAMIENTO DE LAS PRESAS DE ALCALÁ DEL RIOO, CANTILLANA Y PEÑAFLOR. COMPARACIÓN ENTRE EL ÁREA INUNDADA EN 1963 (5.700 m3/s) Y LAACTUAL (<3.500 m3/s)

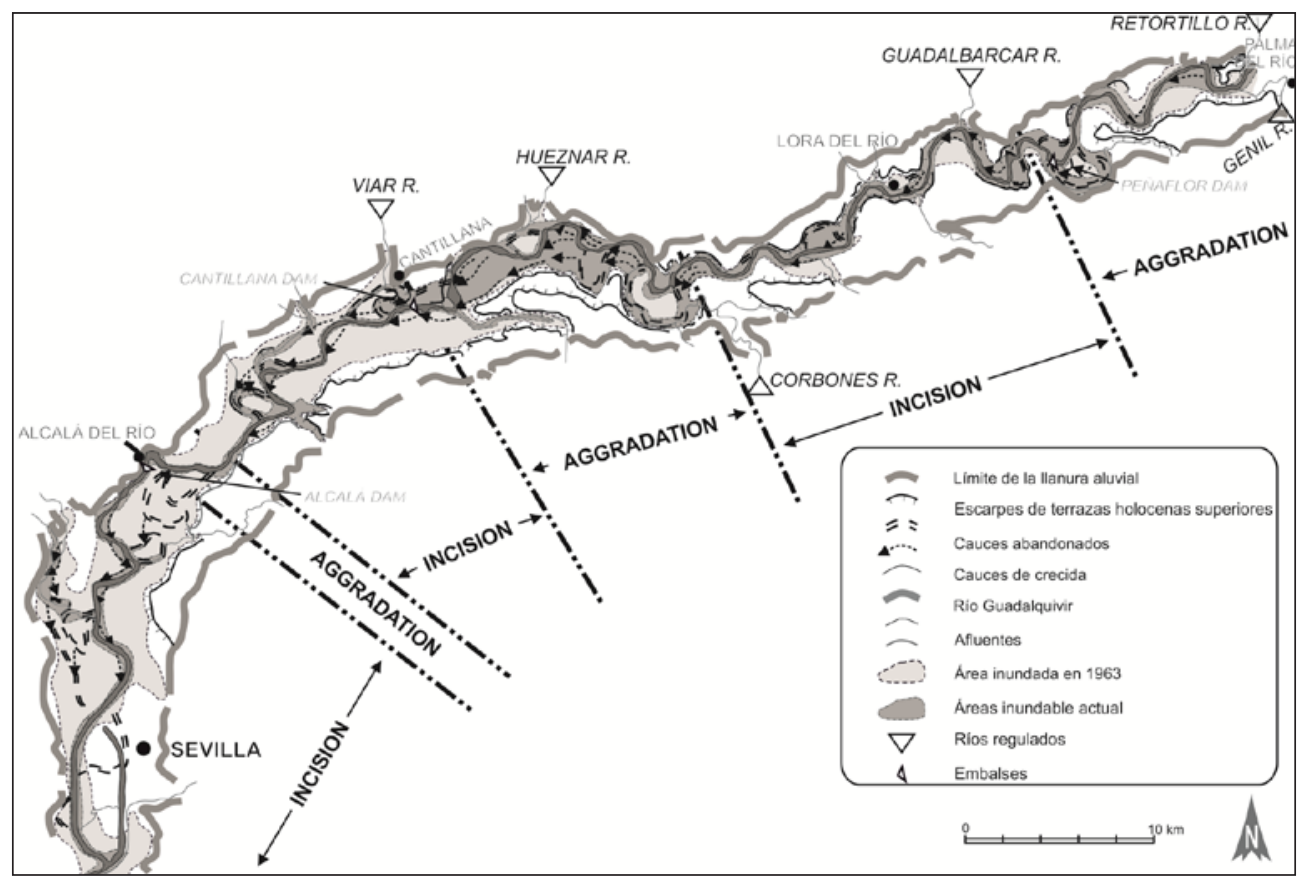

Fuente: elaboración propia.

Junto a ello, los numerosos embalses construidos en los afluentes han alteraron tanto el régimen hidrológico (disminución de los caudales máximos y desaparición de los estiajes) como el tránsito sedimentario. De igual modo, la extracción de áridos de las orillas y el fondo del río favorecieron la reactivación de los procesos erosivos. Estos también se identifican en los afluentes, donde la falta de actividad hidrológica provocó el fuerte desarrollo de la vegetación de ribera que progresivamente ha ido invadiendo los cauces durante los últimos 30 años.

En el segundo caso, en la llanura aluvial, aumentó el riesgo potencial de inundación debido a la alteración o, en muchos casos, total desaparición de los elementos morfohidrológicos imprescindibles para su correcto funcionamiento. Así, el espacio disponible en todo el valle para la laminación de futuras crecidas extraordinarias quedó aminorado e interferido por las numerosas infraestructuras construidas. Éstas han reducido al mínimo las posibilidades de circulación de las aguas fuera del cauce ordinario, creándose potenciales tapones hidráulicos (Sevilla, La Algaba, La Rinconada, Brenes, Cantillana, Tocina, Alcolea, Lora del Río). Así se puso de manifiesto en las crecidas de los años 1996 (Diciembre) y 1997 (Enero y Diciembre) con caudales máximos de entre 3.200 y 3.670 $\mathrm{m}^{3} / \mathrm{s}$ en el aforo de Alcalá del Río, generándose una mayor elevación de la lámina de agua 
por obstrucción y la desviación de los flujos desbordados en el sector comprendido entre Cantillana y Palma del Río. Se alcanzó así una altura y extensión del área inundada similar a la de 1963 en esa zona (Fig. 4), pero con algo menos de la mitad de caudal (Guerrero y Baena, 1998). Por el contrario, aguas abajo de Alcalá del Río y alrededores de la ciudad de Sevilla, estas crecidas no tuvieron efectos destacables ya que funcionó con éxito el sistema estructural de defensa contra inundaciones.

\section{URBANISMO Y NUEVOS RIESGOS DE INUNDACIÓN EN EL SIGLO XXI}

La sensación de seguridad generada en Sevilla durante estas últimas crecidas del siglo XX junto con la especulación del suelo, ha impulsado en el comienzo del nuevo siglo políticas de expansión urbanística por parte de los ayuntamientos más próximos a la ciudad. Y ello, a pesar de que la población de la llanura en 2011 (900.000 hab.), sólo se había incrementado en un 14\% respecto a la existente en la década de los 90 (Instituto de Estadística y Cartografía de Andalucía, 2011).

A esta situación le acompañó el correspondiente aumento de las infraestructuras territoriales que, sin ser numerosas, incrementan aún más la interferencia en la dinámica fluvial. Por un lado, las de carácter defensivo ubicadas en el inicio del tramo de estudio (Fig. 3, 2011), tales como:

a) La carretera de circunvalación sobre muro de defensa contra inundaciones construida al noreste de Palma del Río (A-453) que enlaza con el nuevo puente atirantado sobre el Guadalquivir (2008).

b) El muro de contención contra inundaciones con vial de circunvalación sobre él (A-457), construido al sureste de Lora del Río (Noviembre 2011). Obra de carácter urgente ejecutada tras las avenidas de diciembre-enero de 2009-2010 y febrero-marzo de 2010 .

Y por otro, las ubicadas en las proximidades de Sevilla y destinadas al equipamiento territorial (Fig. 3, 2011):

a) Viales sobre-elevados sobre rellenos de $3 \mathrm{~m}$ de altura respecto a la llanura de inundación destinados a la conexión urbanística entre los núcleos de La Rinconada y San José (2010).

b) Muro de defensa acompañado de relleno para ampliación urbanística y paseo fluvial en La Algaba (2009).

c) Vial metropolitano sobreelevado que integra el nuevo «Acceso Norte a Sevilla» A-8009 (2008).

d) El acondicionamiento ambiental y reforestación de las riberas del río Guadalquivir en la ciudad de Sevilla en su margen izquierda (Parque del Charco de la Pava), con nuevo muro de defensa contra inundaciones inmediato al río y adelantado más de 100 $\mathrm{m}$ respecto al existente en el s. XX (2011).

e) La nueva esclusa de acceso al Puerto de Sevilla como prolongación y ampliación hacia el sur del dispositivo de defensa tradicional (2010). 
Respecto a los embalses se construyen ahora dos más en los afluentes de la margen derecha del Guadalquivir, ríos Guadiato y Viar. El primero denominado «Breña II» $\left(823 \mathrm{Hm}^{3}\right)$ destinado al riego agrícola y el segundo, «Melonares» $\left(185 \mathrm{Hm}^{3}\right)$, para el abastecimiento urbano de la ciudad de Sevilla y su área metropolitana. Pese a este aumento en la capacidad de almacenamiento global de la cuenca estimado en $5.593 \mathrm{Hm}^{3}$ (Confederación Hidrográfica del Guadalquivir, 2013), el río sigue sin estar regulado en su totalidad adquiriendo cada vez mayor importancia el sistema de control en las descargas de urgencia. Así se puso de manifiesto durante las crecidas de diciembre de 2009 y marzo de 2010 o las de diciembre de 2010 y enero de 2011, asociadas a las descargas preventivas de los embalses $\left(2.000 \mathrm{~m}^{3} / \mathrm{s}\right.$ en menos de $24 \mathrm{~h}$ ), alcanzando puntas de crecida de $3.300 \mathrm{~m}^{3} / \mathrm{s}$ como máximo (Agencia Andaluza del Agua, 2011). Estos caudales, aún siendo de escasa magnitud, sorprenden por la extensión y altura que alcanzó la lámina de agua durante la inundación del sector comprendido entre Cantillana y Palma del Río, repitiéndose la situación de 1996. La explicación reside en la coincidencia de una serie de factores en ese tramo como son: de un lado, el incremento en el grado de colmatación del cauce aguas arriba de la presa de Cantillana, donde no se ha efectuado ningún dragado de mantenimiento desde su construcción (Fig. 5); y de otro, la confluencia de los principales tributarios regulados (ríos Genil, Bembézar, Guadalbarcar, Corbones, Retortillo, Huéznar y Viar). Junto a ello, la invasión de la llanura de inundación por nuevas edificaciones amparadas por la administración (P.G.O.Us.), ha incrementado la vulnerabilidad de ciertos núcleos urbanos (Lora del Río, Cantillana, Tocina) ante este fenómeno. Por el contrario en el tramo entre Alcalá del Río y Sevilla, las labores de dragado del río para la navegación desde la desembocadura hasta el puerto continúan favoreciendo la incisión del cauce, facilitan la evacuación hídrica y acrecientan, aún más, la presión urbanística sobre el escaso espacio libre de la llanura de inundación que aún queda en la zona.

Figura 5

COLMATACIÓN POR SEDIMENTOS FINOS DEL CAUCE DEL RIOO GUADALQUIVIR AGUAS ARRIBA DE LA PRESA DE CANTILLANA.

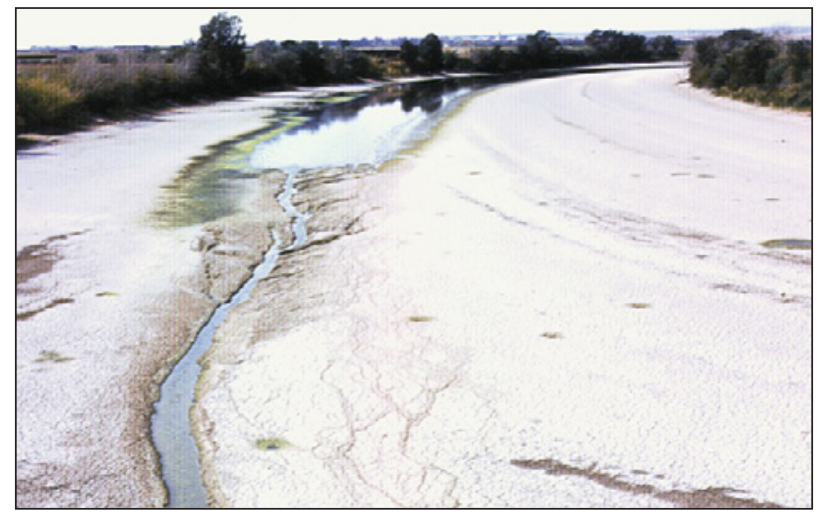

Se aprecia la escasa sección hidráulica del cauce ordinario. Imagen tomada durante la sequía de inicios de la década de los 90 (fecha y autor: septiembre de 1995, Rafael Baena).

Shown the small section hydraulic of the ordinary channel. Picture taken during drought at the beginning of the ninenties of the 20th century (date \& author: september 1995, Rafael Baena). 


\section{CONCLUSIONES}

La extensión y variedad de elementos morfohidrológicos de la llanura aluvial del Guadalquivir, obedece a una dinámica natural vinculada a crecidas extraordinarias y de recurrencia secular. Bajo estas condiciones el hidrosistema había funcionado eficientemente hasta el siglo XVIII-XIX, cuando los problemas de navegabilidad del cauce junto con el incremento en el número de inundaciones, comienzan a modificar la dinámica fluvial. No obstante, el gran momento de transformación generalizada de la llanura se produce en la segunda mitad del siglo XX, asociado a las rectificaciones de trazado y a la regulación de la cuenca hidrográfica que cuadruplica su capacidad de almacenamiento. Esta situación conlleva una falsa percepción de seguridad que favoreció la mayor ocupación urbana y de infraestructuras de la llanura hasta la actualidad. Con ello, elementos morfohidrológicos de gran importancia en el funcionamiento de las crecidas cambian o desaparecen. El cauce ordinario ha reducido su anchura de manera general pero con una dinámica de incisión-colmatación diferenciada y vinculada al dragado y rectificación del cauce para la navegación hasta Sevilla, la ubicación de las tres presas existentes en el río y el predominio de la carga en suspensión. Los cauces abandonados se han colmatado rápidamente, desapareciendo en unos casos bajo las infraestructuras y los núcleos urbanos, o conservando una reducida funcionalidad cuando son ocupados para la agricultura. Los afluentes principales se han visto alterados en su funcionamiento tanto a nivel de caudales como de carga sedimentaria, estando su dinámica condicionada por el control de las presas. Respecto a las terrazas holocenas e históricas han perdido su función de límite de inundabilidad y de laminación de crecidas, no suponiendo hoy en día mayor obstáculo a la circulación de las aguas que cualquier otra intervención humana.

En el siglo XXI, lejos de afrontar la situación anterior mediante una política de planificación territorial e integrada para la cuenca, se ha optado por soluciones parciales y localizadas en aquellos puntos de mayor urgencia ante las inundaciones. Así en las proximidades de Sevilla, las intervenciones principales han consistido en la ampliación del perímetro de muro contra inundaciones, acompañada de una especulación urbanística que, prácticamente, completa el estrangulamiento de la llanura de inundación. Al mismo tiempo hacia el norte de la ciudad (sector La Algaba-La Rinconada), se está repitiendo el mismo modelo de ocupación urbana con la expansión de estos municipios, la construcción de vías rápidas y nuevos muros defensivos. Algo parecido ocurre en Lora y Palma del Río, con obstrucciones parciales de la llanura.

A nivel general, los principales cambios geomorfológicos detectados en el cauce y en el área inundable, se vinculan a un comportamiento diferencial en la tendencia a la incisión o a la colmatación del cauce por sectores. Así, aguas abajo de Alcalá del Río el Guadalquivir presenta una incisión de algo más de $9 \mathrm{~m}$ que le confiere una dinámica de canal hidraúlico para una evacuación óptima de caudales de alrededor de unos $3.500 \mathrm{~m}^{3} / \mathrm{s}$. Por contra, en el sector Cantillana-Alcolea el Río, debido a la fuerte colmatación del cauce aguas arriba de la presa y a la convergencia de descargas de emergencia en los afluentes, estos caudales, sin ser extraordinarios, superan el estado de bankfull y ocupan la llanura de inundación en su totalidad. Ello se ve agravado, además, por el efecto «cascada» de los desembalses de los afluentes, cuando éstos superan su límite de seguridad tras períodos de lluvias regionales intensas. El resultado es un riesgo de inundación muy bajo para la ciudad de Sevilla mientras que 
aguas arriba, con magnitudes de este orden, el riesgo se ha visto considerablemente incrementado. Para evitarlo se ha optado por intervenciones locales mediante muros de defensa y no por una planificación integral, lo que traslada el problema a otros sectores.

La incertidumbre se plantea ante la posibilidad de que se produzca una crecida de mayor magnitud, como la de 1963. En este hipotético caso, los daños serían desproporcionados con una mayor altura de la lámina de agua, el desvío y aceleración de los flujos hídricos desbordados y la inundación de nuevas zonas urbanas, sobre todo al norte de la ciudad de Sevilla.

\section{AGRADECIMIENTOS}

Este trabajo ha contado con financiación procedente de los Proyectos del Plan Nacional de I+D+i 2000-2003, OPN-Ministerio de Fomento (FOM/2003/02), del Excmo. Ayuntamiento de Sevilla «Estudio de programas de actuación en relación con el río Guadalquivir en el término municipal de Sevilla» (SUB-AYUNT/010-05) y de los Contratos arts. 68/83 LOU «Riesgos de inundación en el sector sur de la población de Lora del Río (Sevilla)» (SI096/26) y «Evaluación de la colmatación del río Guadalquivir aguas arriba de la presa de Cantillana (Sevilla)» (FIUS-2338/0741).

\section{BIBLIOGRAFÍA}

AGENCIA ANDALUZA DEL AGUA (2011): Sistema de Información Hidrológica de la Cuenca del Guadalquivir (SAIH). Reference on-line (date: 20/06/2012). Junta de Andalucía. BAENA ESCUDERO, R. (1993): Evolución cuaternaria (3M.a) de la Depresión del mediobajo Guadalquivir y sus márgenes (Córdoba y Sevilla). Tesis Doctoral inédita. Universidad de Sevilla, $589+$ cart.

BAENA, R., GARCÍA, B., GUERRERO, I., HARTVICH, F., LEICHT, B. y MARCHIOL, L. (2004): «Floods 2002 in Prague: reflections, lessons and ideas». Geografie-Sborník $C G S, \mathrm{n}^{\circ} 109,170-180$.

BAENA ESCUDERO, R. (2006): «Los Ríos Como Oportunidad para la Recuperación del Patrimonio Natural de las Ciudades desde la Cooperación Institucional» en Ríos y Ciudades Europeas: Espacios Naturales, Culturales y Productivos. Secretariado de Publicaciones de la Universidad de Sevilla, 37-44.

BAENA ESCUDERO, R., GUERRERO AMADOR, I. y JANSKÝ, B. (2006): «Comparative Analysis of the Floods in Prague (Czechia) and in Seville (Spain): Seen From the Geographical Viewpoint». Geografie-Sborník CGS, n 111, 326-340.

BAENA ESCUDERO, R. y GUERRERO AMADOR, I. (2009): «El Registro Geoarqueologico del Sector sur de la Puerta de Jerez (Sevilla): Ultimos 4000 Años en la Sedimentación Fluvial del Guadalquivir» en VII Reuniao Do Quaternário Ibérico/VII Reunion del Cuaternario Ibérico, Vol. 1 (Boski, T; Moura, D \& Gomes, A. editores.). Faro, Portugal, 175-180.

BAENA ESCUDERO, R., FERNÁNDEZ CARO, J.J., GUERRERO AMADOR, I.C., POSADA SIMEON, J.C. (2014): La terraza compleja del río Guadalquivir en «Las Jarillas» (La Rinconada, Sevilla. SW de España): cronoestratigrafía, industria lítica y macrofauna asociada. Cuaternario y Geomorfología, n ${ }^{\circ} 28$ (3-4), 107-125. 
BESCÓS, A. y CAMARASA A.M. (2004): «La creciente ocupación antrópica del espacio inundable y el aumento de la vulnerabilidad en las poblaciones del bajo Arga (Navarra)». Boletín de la Asociación de Geógrafos Españoles, n 39, 101-117.

BONSOR, G.E. (1931): The Archaeological expedition along the Guadalquivir. 1899-1901. Nueva York. Versión castellana de G. Chic García y A. Padilla Monje (1989). Gráficas Sol, Écija, 133.

BORJA PALOMO, F. (1878): Historia crítica de las riadas o grandes avenidas del Guadalquivir en Sevilla desde su reconquista hasta nuestros días. Reedición del Área de Cultura y Fiestas Mayores (2011). Ayuntamiento de Sevilla. Sevilla, 2t.

BRAVARD, J.P. (2004): «Le façonnement du paysage fluvial de Lyon: choix urbanistiques et héritages hydro-morphologique». Boletín de la Asociación de Geógrafos Españoles, $\mathrm{n}^{\mathrm{o}} 39,17-32$.

BRAVARD, J.P. (2006): «Rivers as cultural and meeting points some reasons for success» en Ríos y Ciudades Europeas: Espacios Naturales, Culturales y Productivos. Secretariado de Publicaciones de la Universidad de Sevilla, 27-35.

BRIERLEY, G. \& FRYIRS, K. (2004): Geomorphology and river management. Applications of River Styles Framework. Blackwell Publishing, England, 416.

CHIC GARCÍA, G. (1979): «Gades y la desembocadura del Guadalquivir». Rev. Gades, 3,7-23.

CHIC GARCÍA, G. (1990): La navegación por el Guadalquivir entre Córdoba y Sevilla en época romana. Gráficas Sol, Écija, 104.

CONFEDERACIÓN HIDROGRÁFICA DEL GUADALQUIVIR (2012): Memoria de Actividades 2009/2011. Ed. Ministerio de Agricultura, Alimentación y Medio Ambiente-Confederación Hidrográfica del Guadalquivir, 147.

CONFEDERACIÓN HIDROGRÁFICA DEL GUADALQUIVIR (1977): Guadalquivires, 50 aniversario. Jerez de la Frontera, Cádiz, 599.

CONFEDERACIÓN HIDROGRÁFICA DEL GUADALQUIVIR (2013): Evaluación preliminar del Riesgo de Inundación en la Demarcación Hidrográfica del Guadalquivir. Documento 1. Ministerio de Agricultura, Alimentación y Pesca. Sevilla, 66.

GARCÍA MARTÍNEZ, B. (1996): Los meandros del río Guadalquivir en su tramo Bajo continental (Palma del Río-Brenes): cambios recientes y evolución geomorfológica. Trabajo de Investigación de Doctorado en Geografía Física. Universidad de Sevilla, 217 + cart. (inédito).

GARCÍA MARTÍNEZ, B. y BAENA ESCUDERO, R. (1997): «Cambios Históricos en la Hidrología del Guadalquivir y su Repercusión en el Meandro de Tocina (Sevilla)», en Cuaternario Iberico. Ed. AEQUA, Huelva, 368-371.

GARCÍA MARTÍNEZ, B., GUERRERO AMADOR, I. y BAENA ESCUDERO, R. (1999): «La dinámica de meandros durante el Cuaternario reciente en la conformación de la llanura aluvial del Bajo Guadalquivir, aguas arriba de Sevilla», en Avances en el Estudio del Cuaternario Español. Gerona, 119-124

GARCÍA MARTÍNEZ, B. (2003): «Intervención paleohidrológica (SS.XVI-XX) del Tramo Bajo continental del río Guadalquivir a través de sus inundaciones y meandros», en Geografía de Andalucía, Ed. Hespérides, 173-213. 
GARCÍA MARTÍNEZ, B. y BAENA ESCUDERO, R. (2006): «El Impacto de las Infraestructuras de la Ciudad de Sevilla Sobre el Paisaje Fluvial del Río Guadalquivir», en Ríos y Ciudades Europeas: Espacios Naturales, Culturales y Productivos. Secretariado de Publicaciones de la Universidad de Sevilla, 119-128.

GARCIA MARTÍNEZ, B. y BAENA ESCUDERO, R. (2008): «El doble meandro abandonado del Guadalquivir en Cantillana (Sevilla): cambios de trazado y evolución geomorfológica». Geographicalia, ${ }^{\circ}$ 53, 101-119.

GARCÍA RUÍZ, J.M. y PUIGDEFABREGAS, J. (1995): «Efectos de la construcción de pequeñas presas en cauces anastomosados del Pirineo central». Cuadernos de Investigación Geográfica, $\mathrm{n}^{\mathrm{o}}$ 11, 91-102.

GARZÓN, G., ALONSO, A., LÓPEZ, J. y ARCHE, A. (1990): «Desbordamiento en el río Jarama (provincia de Madrid) e interferencia humana en la llanura de inundación»,en $I V$ Reunión Nacional Geológica Ambiental y Ordenación del Territorio, Gijón, 259-270.

GAUTIER, E. (1994): «L'Interferences des facteurs antrhropiques et naturels Dans les processus d'incision sur une rivière alpine. L'exemple du Buëch. Alpes du Sud». Revue de Géographie de Lyon, 1, 57-62.

GUERRERO AMADOR, I. y BAENA ESCUDERO, R. (1998): «La inundación del Guadalquivir en diciembre de 1996 (Sector Alcolea del Río-Cantillana, Sevilla)», en Investigaciones recientes de la Geomorfología española (Gomez Ortiz, A; Salvador Franch, F. eds.). Geoforma Ediciones, Barcelona, 203-210.

GUERRERO AMADOR, I., GARCÍA MARTÍNEZ, B. y BAENA ESCUDERO, R. (2014): «Crecidas históricas, transformaciones territoriales y riesgo actual de inundación en la ciudad de Sevilla», en Sevilla, la ciudad y la riada del Tamarguillo (1961). Universidad de Sevilla. Secretariado de Publicaciones, 247-267.

IBISATE, A. (2006): «El río Zadorra a su paso por la ciudad de Vitoria-Gasteiz: el anillo verde y el problema de las crecidas fluviales», en Ríos y Ciudades Europeas: Espacios Naturales, Culturales y Productivos. Secretariado de Publicaciones de la Universidad de Sevilla, 107-112.

INSTITUTO DE ESTADÍSTICA DE ANDALUCÍA (1992): Evolución de la Población. Andalucía 1900-1991. Junta de Andalucía. Sevilla, 155.

INSTITUTO DE ESTADÍSTICA Y CARTOGRAFÍA DE ANDALUCÍA (2011): Sistema de Información Multiterritorial de Andalucía (SIMA). Consejería de Economía, Innovación, Ciencia y Empleo. Junta de Andalucía. Sevilla, CD.

KLINGEMAN, P.C.; BRAVARD, J.P. y GIULIANI, Y. (1994): «Les impacts morphodynamiques sur un cours d'eau soumis à un aménagement hydroélectrique à derivation: le Rhône en Chautagne (france)». Revue de Géographie de Lyon, n ${ }^{\circ}$ 69-1, 73-87.

LANGHAMMER, J \& VILIMEK, V. (2006): «Present approaches to evaluation of anthropogenous changes in landscape as a factor of flood risk». Geografie-Sborník CGS, n 111 , 233-246.

MACKLIN, M.G. \& LEWIN, J. (2003): «River sediments, great floods and centennial-scale Holocene climate change». Journal of Quaternary Science, $\mathrm{n}^{\circ}$ 18, 101-105.

MASACHS, V. (1954): Las aguas. Geografía de España y Portugal. Ed. Montaner y Simón. Barcelona, tomo 2, 80-142. 
MINISTERIO DE OBRAS PÚBLICAS Y URBANISMO (1985): Estudio de inundaciones históricas. Mapa de riesgos potenciales. Cuenca del Guadalquivir. Dirección General de Obras Hidraúlicas. Madrid, t. II, 524.

MINISTERIO DE OBRAS PÚBLICAS Y URBANISMO (1991): Inventario de presas españolas. Dirección General de Obras Hidraúlicas. Madrid, t. II, 524.

OLLERO OJEDA, A. (1991): Estudio ecogeográfico de los meandros del Ebro en el sector Rincón de Soto-novillas. Ministerio de Obras Públicas y Urbanismo, 334 pp.

PEIRY, J.L. et NOUGUIER, F. (1994): «Le Drac dans l'aglomeration de Grenoble: première evaluation des changenments géomorphologiques contemporains». Revue de Géographie Alpine, $\mathrm{n}^{\mathrm{O}} 82-2,77-96$.

PONSICH, M. (1974): Implantation Rural Antique sur le Bas-Guadalquivir I. Publications de la Casa de Velàzquez, Série Archéologie II, Madrid/Paris, 404.

RAMOS, A. (1983): Los mecanismos y los procesos de inundación en la ciudad de Alicante. Lluvias torrenciales e inundaciones en Alicante, Instituto Universitario de Geografía. Alicante, 73-98.

ROUX, A. L.; BRAVARD, J.P; AMORÓS, C. \& PATOU, G. (1989): «Ecological changes of the French upper Rhône River since 1750», en Historical changes of large alluvial rivers: western Europe (Petts, G. Editores), John Wiley \& Son, Chichester, 323-350.

SCHUMM, S.A. (1977): The fluvial system. John Wiley and Sons, London, 338.

TOCKNER, K. \& STANFORD, J.A. (2002): «Riverine flood plains: present state and future trends». Environmental Conservation, $\mathrm{n}^{\circ} 29,308-330$.

VANNEY, J.R. (1970): L'Hydrologie du Bas Guadalquivir. C.S.I.C., Inst. Geografía Aplicada. Madrid, $176 \mathrm{pp}$.

WARD, R. (1978): Floods: A geographical perspective. MacMilan, London, 224 pp. 
